\title{
User Centered Inclusive Design for People with Dyslexia: Experiences from a Project on Accessibility
}

\author{
Dominik Rupprecht, Rainer Blum, and Birgit Bomsdorf \\ University of Applied Sciences, Department of Applied Computer Science, Fulda, Germany \\ \{dominik.rupprecht, rainer.blum, \\ birgit.bomsdorf\}@informatik.hs-fulda.de
}

\begin{abstract}
User participation is a key element in user centered design of interactive systems. However, applying established methods is not straightforward while realizing a system for people with cognitive impairment due to their specific, heterogeneous needs and abilities. This paper presents experiences from conducting a user centered inclusive design process within an ongoing project. It aims at the development of a web application for people with dyslexia caused by cognitive impairment. A distinctive feature is the intended use of the application: It must support the counseling interviews for planning the real life inclusion of the target group. In addition, it must enable cognitively impaired as well as non-impaired people using it cooperatively. One of the most challenging issues was how and when to involve cognitively impaired users.
\end{abstract}

Keywords: User Centered Design, Inclusive Design, Accessibility, User Participation, Methods and Techniques, Dyslexia.

\section{Introduction}

In user centered design (UCD) the focus throughout the development process is on the users' needs and (dis-)abilities. Applying traditional methods, however, is not straightforward while realizing a system for people with cognitive impairment. For example, most of the well-known methods of user participation, a key element in UCD, rely on face-to-face meetings that are not easily applicable, because of a lack of knowledge of the diverse, heterogeneous characteristics of the user group. Furthermore cognitively impaired may be unable to concentrate on a topic or to utter a want.

All in all, designing interactive systems for and with people with disabilities and their involved personal supporters (caregivers, relatives) is highly complex because of the resulting multi-faceted interplay of needs, abilities, and objectives. Involving people with disabilities in the design process, even though challenging, is important to increase the understanding about these complexities and enables to create more meaningful and useful systems for them. We adapted in a current project, similar to Newell et al. [1], traditional UCD methods aiming at a user centered inclusive design process. This contribution presents lessons learned, particularly in facing the challenge of involving people with cognitive impairment - and additional stakeholders. 
As the focus is on UCD methods, usability problems are only mentioned but not reported in detail.

In the remaining part of this section the project background is introduced and related work is reported. Section 2 shows the involvement of the clients throughout the project. The third section outlines the usage of guidelines for the target application. The final section gives a summarization and a short outlook on ongoing work.

\subsection{Background of the Project}

The main objective of the project referenced in this paper is the investigation of how interactive web forms can be accessible for people with dyslexia caused by cognitive impairment. The proof-of-concept example is a specific form intended to discuss and plan central aspects of the daily life of individuals with impairments. This form is used once a year in collaboration with the concerned persons to perform counseling interviews. The aim is to determine required help and integration services. In these interviews information on the current situation, impairments and wishes of the impaired people are collected. This builds the basis for granting vital support activities in the areas of self-care, living and working for the next months. The currently used form is a PDF document of eight pages crowded with arrays of questions. During the interviews it is used by assistants not only to collect the needed data, but to keep track of the whole process.

Three different stakeholders are involved in the project: The first user group in focus are people with dyslexia attributable to cognitive impairments, in the following referred to as "clients". The second group comprises caregivers and relatives of the clients guiding the counseling interviews, referred to as "assistants". The last group consists of the marketer of the form (the current PDF form and the upcoming web application), a small-sized enterprise with years of experience in providing trainings for using the form.

\subsection{Related Work}

McCarthy and Swierenga [2] give an overview of the literature considering dyslexia and accessibility produced until 2010. The authors highlight that much work has been done in the topics of dyslexia and web accessibility separately. But there is only a small amount of work addressing both aspects at once. They also report that most efforts are focused on how to support users that are blind or have low vision.

De Santana et al. [3] give a good and comprehensive overview over existing guidelines regarding people with dyslexia. They present 41 guidelines to support three website stakeholders: Developers, designers and content producers. These guidelines were gathered on websites discussing dyslexia and accessibility and are based on available sets of guidelines like the Web Content Accessibility Guidelines (WCAG) [4]. The authors divided the guidelines in the following groups: navigation, colors, text presentation, writing, layout, images and charts, end user customization, markup, and videos and audios. One conclusion the authors draw is that end user customization is an important step to achieve accessibility considering dyslexia. But they identified only two guidelines and recommended a deeper study on end user customization. 
The Web Accessibility Initiative (WAI) [5] of the W3C proposes an analysis procedure when working with guidelines containing three phases: evaluation by experts, automatically or manual conformity assessment and user evaluation. For the conformity assessment the WebAIM [6] project created a checklist (Cognitive Web Accessibility Checklist) and the self-acting evaluation tool WAVE [7] based on the WCAG guidelines.

Newell at al. [1], [8] point out the need for a shift of paradigm towards a "User Sensitive Inclusive Design" extending the methodologies of the UCD. The authors show challenges when working with people with disabilities and point out differences to the UCD. Challenges we identified also in our project are:

- Users may not be able to communicate their thoughts,

- Users may not be the purchaser of the final product,

- Users with disabilities may have specialized and little known requirements and

- Different user groups may provide very conflicting requirements for a product.

Differences we also identified in our project are:

- Much greater variety of user characteristics

- Difficulty of finding and recruiting "representative users"

- Conflicts between accessibility and ease of use for less disabled people

- Possible conflicts of interest between accessibility for people with different types of disabilities

Existing methods and approaches are a good starting point to support the work with disabled users. Newell et al. [1] points out that the designer should develop an "empathetic relationship" with disabled users, rather than rely on standards and guidelines. Thus, following the idea of participatory design, the authors underline the importance of the involvement of the impaired users from the beginning. Furthermore, they suggest to begin the design process with only a narrow list of features to get products that are usable by the target user group and to avoid function-overloaded products the users cannot work with.

They also argue for the use of personas in the early project phases. Personas are descriptions of hypothetical archetypes of actual user groups and are developed based on identified user profiles. Zhang et al. [9] make use of personas in the early design and also in the evaluation phases. In their methods, called "persona self-guided evaluation method" and "persona think-aloud method", experts act from the perspective of the persona. These methods cannot fully replace user testing, but enable designers to create a better product when the target user population is not accessible. Working with personas can be helpful to avoid ethical and legislative barriers in working with impaired people. Another approach to this is proposed by Newell et al. [1]: Instead of asking experts to adopt the roles of personas they had well-briefed professional actors working to a well-crafted script in the phase of design. The actors provided a personification of the target user and throughout improvisation interacted with the designers or the users. The authors showed that this method can be a powerful tool of communication between designers and users of technology and in raising the designers' awareness of the challenges when working with disabled people. 
There are some projects working with people with dyslexia or cognitive impairments, too. For example Sutcliff et al. [10] investigated an e-mail system for users with cognitive impairments (e.g. attention span problems, working memory limitations, mild problem-solving and language disorders). They showed that with cognitively impaired people, often, training is no solution when there are problems with the user interface. Or Hagelkruys et al. [11] who are working on the ongoing LITERACY web portal to improve social inclusion of youth and adults with dyslexia. They use additional tools like screen reader to support people with dyslexia. The usage of those tools is not part of this paper, but is considered in our development.

\section{Involvement of Clients}

At the beginning of the project all members of the project consortium assumed that substantial contributions of the clients for the analysis could not be expected due to their cognitive and verbal impairments (compare to [8]). Within the early phase of gathering requirements only assistants and the marketer were involved but no clients. However, members of the development team visited six clients in their daily environment like the foster homes they are living in to get a better understanding of the target group (compare to [1]). Out of their observations and statements of the caregivers different client profiles were built containing information about their computer skills like Internet usage, their form of dyslexia and additional physical impairments. This was the basis for building personas to be used at least in the first development and evaluation steps.

During the process the importance to get information from both the clients and the caregivers was underlined and increased. On the one hand, more skills of the clients were observed than the caregivers knew of. On the other hand, the evaluation recordings were analyzed together with caregivers, because the clients have learned to pretend and fake social interaction skills - thus some of their impairments were not visible at first glance.

Early on in the first four months of the project it became clear that the clients could be involved more often. So the project consortium decided to shorten the planned development cycles and to include the clients directly in the process instead of the personas ahead of the original schedule.

To get a greater user base in order to prevent the need of redesign in later phases of the project the initial group of six clients was extended over the course of the project up to eleven clients. With each extension the range of client profiles grew based on the additional information and the existing personas had to be altered and additional ones had to be created. For example, all clients from the beginning were familiar to the web and to browsers. Subsequently, clients without these abilities joined in, resulting in a very heterogeneous group of clients. With each addition more assistants joined in, too. All of the clients were familiar to the counseling interviews and the original PDF form. Not only in the meetings with only the assistants and the marketer but in general each of them favored a different group of clients. Hence it was harder to come up with coherent solutions, and from time to time the focus was on a subgroup but mostly the design considered the whole group. It is still challenging to specify priorities of user profiles or of impairments to lead the design processes. 
Prototypes of different fidelity and type were utilized throughout the development process. As also suggested by Zhang et al. [9] we started with sketches and paper prototypes to come up with a number of different ideas. Based on selected solutions simple HTML pages were created including only parts of the intended system. Later on we used interactive prototypes using the wireframe and rapid prototyping tool Pidoco [12]. It enabled us to realize interactive low-fidelity prototypes very fast to get feedback on them and to evaluate those involving assistants and the marketer.

Even the first evaluations were conducted with the clients and not by using personas as projected before. This decision resulted from the positive experiences. The evaluations with the clients were conducted as field tests taking place in their familiar surroundings. They were performed in form of a user test combined with further standard usability methods like questionnaires and scenario-based tasks the user were asked to perform as well as audio and video recording. The recordings were analyzed together with the caregivers and assistants so that they could individually interpret the reactions and statements of the clients.

For the different client groups nearly identical scenario-based tasks and test settings were implemented. We started with the first HTML prototype with only limited functionality to check the hierarchy, navigation structure and simple input fields. The clients understood that the prototype did not represent the whole PDF form they are familiar with and no problems with this artificiality of the system were observable. Whether or not the clients realized the artificial situation became not clear, but this was not a problem, too. But quickly we realized that the process of filling out the PDF form is really essential to the life of the clients. The clients were enthusiastic being part of the test and started to work on their participation in life. For example, they were not in favor to fill in "only" test data but wanted to have their actual data filled in. Therefore, a person of the evaluation team had to assist them by instructing them how to type single words. In subsequent evaluations we considered bigger parts of the counseling interviews and followed the known counseling procedure.

With each of the conducted four iterations the range of functions and the level of detail of the implementation grew resulting in an evolutionary prototyping with a fully functional demonstrator version of our application at the end.

Other variations from the original procedure based on the PDF form were that the clients wandered from one topic to another and didn't focus on the given task. Sometimes the clients only wanted to show what they could do with the computer, regardless if it was on subject or not.

As mentioned above clients are used to hide their impairments and have strategies to avoid attracting attention. This made it difficult to get information from the clients about their opinions of the tested prototypes. Most of the time it seemed that the clients had two strategies: providing answers that they assumed the observers wanted to get or providing answers by which they hoped to avoid follow-up questions. When they were asked to mention positive and negative points about the application, almost all answers were that everything is all right - although problems were observed. Particularly in the first two prototypes clients were not aware of most of the interactive elements and navigation possibilities. However they were not pointed to the problems (afterwards) not to unsettle them and to keep up their motivation to participate. 


\section{Guidelines}

Another part of our approach was consulting existing accessibility guidelines. The summary of de Santana et al. [3] was a good starting point. Typical guidelines like avoiding scrolling, recommended text size or the use of short, simple and direct sentences were considered throughout the design phases. We also used the mentioned WAVE tool [7] to automatically check against the common accessibility guidelines.

Additionally the project consortium committed to advance guidelines. It was discussed which (minimal) mental model of the application the users should be required to build and what the clients are capable of. We wanted to use as few different concepts as possible (compare to [1]) that have to be understood by the clients (easy-ofuse), because training of using the interface was not possible (see [10]).

It was not possible to show the entire hierarchy and structure of the eight-page PDF form at once. The form was therefore split up resulting in a hierarchical structure. According to the assistants' and marketer's previous knowledge about the clients the number of layers of the hierarchy was reduced to four (more width, less depth), each layer containing a set of elements that could be shown only partially. The main question at this point was how to support the clients to move through the hierarchy even if they are unable to build a mental model of it. This could be split up into:

- How to show that there is more to find in lower levels?

- How to show that there is more on the same level?

We re-formulated such questions from the client perspective using simple language (e.g. if at a current subordinate node of the hierarchy a set of elements exists that could not be presented at a time: "How to show, that there is more?" "How to show, that there is a sub-layer?"). This helped us in different ways: Fist it supported the developers to empathize with the clients and to get the perspective of the clients. Second it helped in communicating with the assistants, because this communication can be difficult as they come from different backgrounds and have different jargons (compare to [8]). Further guidelines refer to the visual design of the interface, e.g.:

- No use of 3D elements: A use of three-dimensional elements in the interface was not possible, because this representation would demand too much cognitive resources of the clients to understand and work with it.

Other examples of guidelines and conflicts between them are:

- Prevent distractions: Each webpage should contain only one input field. The attention of the clients should be targeted only at one information unit at a time to prevent distraction. At the same time, however, every option should be visible. Following "out of sight, out of mind" an invisible option will most likely be forgotten. In the current prototype some pages contain more than one input field to preserve information. Additionally, each webpage contains as many visual clues as possible to navigate to the remaining input fields.

- Control: No use of a wizard to guide the users. Some of the assistants and the marketer wanted that the clients are enabled to have free control over the dialog and navigation. The clients should be able to visit the pages in an arbitrary order not to 
force a specific sequence in filling out the form. Later on assistants and marketer agreed with the developers that software assistants such as a wizard are needed.

- Always save automatically: The clients do not have to save their inputs explicitly, e.g., by a "Save"-UI-Element. The behavior of the application should be like filling out a paper based form, where you do not need to save the inputs separately. This was in conflict to the assistants' expectation, who were missing the explicit and familiar saving function when working with the application.

General applicability of these guidelines is still under investigation. Nevertheless, these guidelines often served us within the project to find a common sense. Every time an additional feature was under discussion, it was counter-checked to the guidelines. For example, based on their own "prevent distraction" guideline the assistants and the marketer were convinced not to demand too much additional functionality per page.

\section{Conclusion and Future Work}

In this paper we presented experiences from adapting UCD for a user centered inclusive design process within a project aiming at the inclusion of people with dyslexia caused by cognitive impairments (clients). The proof-of concept application was taken from the domain of digital forms the clients have to understand and interact with.

One finding is that real clients could be involved much earlier than assumed before. Their contribution to the analysis and evaluation is essential even if this contribution is more difficult to achieve due to the cognitive and verbal impairments. In our project the clients were highly encouraged to work with us. They were proud to take part and working with us was a welcome distraction of their daily life. All in all, they asked to be more involved. For the developers this was a very positive experience, too, and they are willing to expand client participation even though it is more challenging and time consuming. Statements and behavior of the clients have to be analyzed very carefully since the clients are used to "fake", i.e. they develop strategies to hide their impairments. Not only because of this but in general, working with the assistants and caregivers is very important due to their specific knowledge and expertise about their clients.

Throughout the project the use of rapid prototyping and of the developed guidelines proved to be valuable to find solutions. The prototypes essentially supported communicating design ideas within the project team (assistants, marketer, and developers). New features were easily counter-checked against the guidelines. The impact on the whole system of features to be implemented was rapidly checked by new prototype versions.

One challenge for the developers was to implement "simple" solutions, e.g. "no use of 3D elements" (see above). However, reactions of the stakeholders to the current system were positive. One of our next step is to evaluate, if the developed application can improve the counseling interviews and thus the inclusion of impaired people. 
Acknowledgement. This project (HA project no. 310/11-55) is funded in the framework of Hessen ModellProjekte, financed with funds of LOEWE - Landes-Offensive zur Entwicklung Wissenschaftlich-ökonomischer Exzellenz, Förderlinie 3: KMUVerbundvorhaben (State Offensive for the Development of Scientific and Economic Excellence).

\section{References}

1. Newell, A.F., Gregor, P., Morgan, M., Pullin, G., Macaulay, C.: User-Sensitive Inclusive Design. Univ Access Inf. Soc. 10(3), 235-243 (2011)

2. McCarthy, J.E., Swierenga, S.J.: What we know about dyslexia and Web accessibility: a research review. Univ. Access Inf. Soc. 9(2), 147-152 (2010)

3. de Santana, V.F., de Oliveira, R., AAlmeida, L.D.A., Baranauskas, M.C.C.: Web Accessibility and People with Dyslexia: A Survey on Techniques and Guidelines. In: Proceedings of the International Cross-Disciplinary Conference on Web Accessibility, W4A 2012, pp. 35:1-35:9. ACM, New York (2012).

4. Web Content Accessibility Guildlines (WCAG) 2.0, W3C Recommendation, (December 11, 2008), http: / / www.w3 . org/TR/WCAG2 0/

5. Web Accessibility Initiative (WAI), http: / / www . w3 . org/WAI /

6. WebAIM - web accessibility in mind, http: / /webaim.org/articles/evaluatingcognitive

7. WebAIM: WAVE - web accessibility evaluation tool, http: / /wave. webaim.org/

8. Newell, A.F., Gregor, P.: User sensitive inclusive design. - in search of a new paradigm. In: Scholtz, J., Thomas, J (eds) Proceedings on the 2000 Conference, Arlington, Virginia, United States, pp. 39-44 (2000)

9. Zhang, H., Hung, Y.-H., Dhillon, G.S., Yang, Y., Mccrickard, D.S.: Social Reader: a Cognitive Stimulation Approach towards Helping Dyslexics. In: International Conference on Kansei Engineering and Emotion Research (2012)

10. Sutcliffe, A., Fickas, S., Sohlberg, M.M., Ehlhardt, L.A.: Investigating the usability of assistive user interfaces. Interacting with Computers 15(4), 577-602 (2003)

11. Hagelkruys, D., Struhár, J., Motschnig, R., Balharová, K.: Including Dyslexic Users in the Early Design of the LITERACY Portal. In: Proceedings of the Iadis International Conference: Interfaces and Human Computer Interaction, pp. 273-277. IADIS, Praha (2013)

12. Pidoco - The Rapid Prototyping Tool, http:/ / pidoco. com 